\title{
A novel UBE2A splice site mutation with intellectual disability type Nascimento
}

Shuyuan Yan ${ }^{1}$, yanling wang ${ }^{1}$, Ying chen ${ }^{1}$, Xiaoni Kuang ${ }^{1}$, Da Hou ${ }^{1}$, Xueyi Li $^{1}$, Linglin Pan $^{1}$, Guangwen Huang ${ }^{1}$, Xiangwen Peng ${ }^{1}$, Jun $\mathrm{He}^{1}$, and Tuanmei Wang ${ }^{1}$

${ }^{1}$ Hunan Normal University

September 7, 2021

\begin{abstract}
Recently, only two splice-site mutations of the UBE2A gene have been observed in patients with X-linked ID type Nascimento (XLID). We found a novel splice site mutation in UBE2A (c.241+1 G>A) and novel clinical appearances, including a typical four-finger line and erected head unstable.
\end{abstract}

\section{Title}

A novel UBE2A splice site mutation with intellectual disability type Nascimento

Author names and affiliations

Shuyuan Yan ${ }^{1}$, Yanling Wang ${ }^{1}$, Ying Chen ${ }^{1}$, Xiaoni Kuang ${ }^{1}$, Da Hou ${ }^{1}$, Xueyi $\mathrm{Li}^{1}$, Linglin $\mathrm{Pan}^{1}$, Guangwen Huang $^{2}$, Xiangwen Peng ${ }^{1 *}$, Jun $\mathrm{He}^{1^{*}}$, Tuanmei Wang ${ }^{{ }^{*}}$

1 Changsha Maternal and Child Health Hospital Affiliated to Hunan Normal University, Changsha 410007, China.

2 Hunan Provincial Maternal and Child Health Care Hospital, Changsha, 410008, Hunan Province, China.

* Corresponding author:

Tuanmei Wang (Email: 1396308863@qq.com)

Jun He(Email: cnhjdoc@126.com)

Xiangwen Peng(Email: pxw1237@163.com)

\section{Abstract}

Recently, only two splice-site mutations of the UBE2A gene have been observed in patients with X-linked ID type Nascimento (XLID). The patients with XLID had similar phenotypes, including speech impairment, severe intellectual disability, hearing loss, wide face, synophrys, generalized hirsutism, urogenital abnormalities. Here, we report a Chinese boy with a clinically very similar syndromic form of XLID, such as speech impairment, severe intellectual disability, moderate hearing loss. However, there are also different characteristics, including erected head unstable, typical four-finger line. Subsequent whole-exome sequencing showed a novel splice site mutation in UBE2A (c.241+1 G>A). Together, not only our study expands the mutation spectrum and clinical characteristics of UBE2A deficiency syndrome (also called XLID), but also this study may provide clinical evidence for genetic diagnosis.

Keywords: UBE2A;X-linked ID type Nascimento; Mutation;Splice site mutation; Whole-exome sequencing;Intellectual disability 


\section{Introduction}

X-linked ID type Nascimento (XLID), characterized by a syndromic intellectual disability due to gene mutation on the $\mathrm{X}$ chromosome, has great attention caused by the high incidence rate of males in intellectual disability [1-5]. According to recent reports, almost 15 percent of the X-chromosome genes that is known to be related to intellectual disability, while only accounts for about 5 percent of the human genome[6]. However, the majority of mutations in XLID genes remain unknown.

Ubiquitin-conjugating enzyme E2 (UBE2A), involved in the proteasome pathway of protein degradation and DNA repair [7], is located on Xq24 [8-10]. UBE2A deficiency syndrome, also known as X-linked ID type Nascimento (MIM \#300860), was first described by Nascimento in 2006, which was characterized clinically by pronounced retardation of psychomotor development, wide face, synophrys, generalized hirsutism, urogenital abnormalities [11]. Since then, two splice-site mutations in UBE2A, seven missense mutations in UBE2A, and four larger deletions in UBE2A have been found [7, 12]. However, there have been fewer reports of UBE2A splice site mutation in china.

Here, we report a Chinese patient diagnosed with XLID, and a novel UBE2A splice site mutation (c.241+1 $\mathrm{G}>\mathrm{A}$ ). In addition to the clinical features of white matter abnormalities in MRI and a recognizable face like wide faces are the same as the typical features reported in XLID. Moreover, the novel clinical features of erected head unstable, no hirsutism, no synophrys, healthy heart, were found. Taken together, the finding of novel mutations of UBE2A in XLID will be better to prevent disability in humans and more possibility to explore the molecular basis of intellectual disability.

\section{Methods}

\section{Ethical compliance}

The written informed consent was obtained from his parents, and all procedures were reviewed and approved by the Ethics Committee of Changsha Hospital for Maternal and Child Health Care Attached to Hunan Normal University (Hunan, Changsha, China).

\section{DNA extraction}

Genomic DNA was extracted from peripheral blood of the proband using DNA extraction kits and standard protocols (TSP201-50, Tsingke, China).

\section{Sanger sequencing}

For analysis of UBE2A splice site mutation, the intron-based exon specific primers were designed with Primer 5 (primer-F: TGGGCCCAGTTTCTTAAGGA; primer-R: AATCAGGAGGCTCCCAGACT). The primers were used for amplify UBE2A exons. PCR was amplified with FastStart Taq DNA Polymerase, dNTPack (Roche) and purified with DNA Gel Extraction Kit ( GE0101-50, Tsingke, China) following standard protocols. BigDye Terminator v3.1 Cycle Sequencing Kit was used for sequencing reactions prior to sequencing on a 48-capillary 3730 DNA Analyzer (Applied Biosystems).

\section{Case presentation}

A 2-years-old male proband (figure 1a, 1b)was diagnosed with severe intellectual disability. However, we did not find chromosome abnormality or the abnormal number of chromosomes. Notably, only one mutation of the patient was found by whole-exome sequencing, which was a novel UBE2A splice site mutation (c.241+1 $\mathrm{G}>\mathrm{A}$ ) (figure $2 \mathrm{a}, 2 \mathrm{~b}$ ). This splice site mutation was not previously reported or annotated in any public databases,and it modified one nucleotide near exon 4 (figure 3a), likely influencing its splicing. Similarly, the splice site mutation was assessed with a pathogenic criterion (PVS1). His parents without mental retardation were examined by whole-exome sequencing, and the results indicated that the boy had inherited 241+1 G>A mutation from his mother (Figure 2a).

The proband was born in the 35th week of gestation with a birth weight of $2150 \mathrm{~g}$ as the first child of a healthy, non-consanguineous couple of Chinese descent. After born by cesarean section due to fetal hypoxia, 
the infant was admitted to the neonatal intensive care unit for 28 days because of hypoxia and premature delivery.according to his parents. Four-dimensional color doppler of gestation age $34+4 \mathrm{w}$ showed stricture of the aortic arch, isthmus narrowing, abnormal shape of the umbilical vein, higher the systolic-diastolic ratio (S/D) of the umbilical artery, poor S/D, and pulsatility index (PI) of the middle cerebral artery. Normal fetal karyotype was examined by using umbilical cord blood.

A male proband at the age of 7 months, was consulted in the Changsha Hospital for Maternal\&Child Health Care after initial symptoms were detected and which were characterized by still erected head unstable and frequent receded head. Thus, he was examined by Gesell's infant development scale score (Gesell) to 12, whereas Gesell's score $<25$ indicates very severe neurological damage [13]. The facial appearance included a wide round face, ocular hypertelorism, short hands and feet, and a typical four-finger line [14] (figure 1a, 1b). Brain magnetic resonance image (MRI) performed abnormal signal changes in the deep white matter area near the lateral ventricle and mild delay of myelination compared to a healthy child at the age of 7 months (Figure 4).

\section{Discussion}

In the present study, we used whole-exome sequencing to identify a novel UBE2A splice site mutation $($ c. $241+1 \mathrm{G}>\mathrm{A})$ in a Chinese proband with an X-linked Intellectual Disability. According to recent reports, this is the second case report of this syndrome with a novel splice site mutation in China.

Notably, we report novel molecular and clinical data of this patient with intellectual disability. Thus far, only two splice-site mutations in UBE2A, seven missense mutations in UBE2A, and four larger deletions in UBE2A have been reported $[7,12,15-23]$. Besides, all probands with UBE2A deficiency syndrome have resemblance phenotypes, including characteristic facial appearance, speech anomalies, and intellectual disability [20, 24]. However, our proband found novel clinical appearance, including a typical four-finger line and erected head unstable, which has not been described in previous patients.

In addition, the phenotypes of all reported proband with UBE2A mutational syndrome and a patient described in the present study were analyzed, three groups of UBE2A mutational syndrome could be classified: (1) the group of those with larger deletions (largedel), (2) the group of those with missense mutation (miss) and (3) the group of those with splice site mutation (splice) (Table 1). All patients were male and showed severe intellectual disability and speech impairment.

Interestingly, the boys with splice site mutation had a significantly higher erected head unstable (EHU) (1/1), white matter abnormalities (WMA) (2/2), wide face (WF) $(4 / 4)$ and small penis (SP) $(2 / 2)$, compared to boys with a missense mutation $(0 / 0 \mathrm{EHU}, 3 / 6 \mathrm{WMA}, 3 / 7 \mathrm{WF}, 8 / 12 \mathrm{SP})$ or larger deletions $(0 / 0 \mathrm{EHU}$, $6 / 7$ WMA, 0/0 WF, 7/9 SP). Furthermore, these results confirmed that splice site mutation of UBE2A significantly decreased the risk of synophrys (2/4), Heart defects (HD) (0/2), Upslanting palpebral fissures (UPF) (0/4), compared with a missense mutation of UBE2A (12/13 synophrys, $3 / 4 \mathrm{HD}, 3 / 8 \mathrm{UPF})$ or larger deletions of UBE2A (6/9 synophrys, 9/9 HD, 7/9 UPF) [7, 11, 18, 19, 22, 25]. According to recent reports, four patients have not been reported with upslanting palpebral fissures. Although these analyses were based on a small statistically significant number of patients that could not get a valid conclusion, it is also worth noting this observation suggested that UBE2A splice site mutation might not be at improved risk for upslanting palpebral fissures and heart defects. Conversely, UBE2A splice site mutation might be at improved risk for wide face, white matter abnormalities and small penis.

In conclusion, this report has demonstrated a novel splice site mutation $(c .241+1 \mathrm{G}>\mathrm{A})$ in UBE2A gene resulting in an aberrant appearance and severe intellectual disability in a Chinese proband. The patient was found novel clinical appearances, including a typical four-finger line and erected head unstable. Together, our report expands the mutation spectrum and clinical characteristics of UBE2A deficiency syndrome (also called XLID) and may provide clinical evidence into genetic diagnosis.

\section{Conflict of Interests}

The authors declare that they have no conflict of interest. 


\section{Acknowledgments}

We thank all the patients and healthy volunteers who agreed to participate in the present study and all those who helped us successfully complete the research.

This work was financially supported by Health Commission of Hunan Province ( B2019138, Y.Y.), in part by Social Development Project of Hunan Province Key Research Plan (2020SK3044, J.H.), and Major Scientific and Technological Projects for collaborative prevention and control of birth defects in Hunan Province (2019SK1010, G.H.). The funders had no role in the study design, data collection and analysis, decision to publish, or preparation of the manuscript.

\section{References}

1. Piton A, Redin C, Mandel JL:XLID-causing mutations and associated genes challenged in light of data from large-scale human exome sequencing . American journal of human genetics 2013, 93 (2):368-383.

2. Lee YR, Khan K, Armfield-Uhas K, Srikanth S, Thompson NA, Pardo M, Yu L, Norris JW, Peng Y, Gripp KWet al : Mutations in FAM50A suggest that Armfield XLID syndrome is a spliceosomopathy . Nature communications 2020,11 (1):3698.

3. Louie RJ, Collins DL, Friez MJ, Skinner C, Schwartz CE, Stevenson RE: Schimke XLID syndrome results from a deletion in BCAP31. American journal of medical genetics Part A 2020, 182 (9):21682174 .

4. Ziats CA, Schwartz CE, Gecz J, Shaw M, Field MJ, Stevenson RE, Neri G: X-linked intellectual disability: Phenotypic expression in carrier females . Clinical genetics 2020, 97 (3):418-425.

5. Pravata VM, Omelkova M, Stavridis MP, Desbiens CM, Stephen HM, Lefeber DJ, Gecz J, Gundogdu $\mathrm{M}$, Ounap K, Joss $\mathrm{S}$ et al : An intellectual disability syndrome with single-nucleotide variants in O-GlcNAc transferase . European journal of human genetics : EJHG 2020, 28 (6):706-714.

6. Neri G, Schwartz CE, Lubs HA, Stevenson RE: X-linked intellectual disability update 2017 .American journal of medical genetics Part A 2018,176 (6):1375-1388.

7. Thunstrom S, Sodermark L, Ivarsson L, Samuelsson L, Stefanova M: UBE2A deficiency syndrome: a report of two unrelated cases with large Xq24 deletions encompassing UBE2A gene . American journal of medical genetics Part A 2015,167A (1):204-210.

8. Shen JD, Fu SZ, Ju LL, Wang YF, Dai F, Liu ZX, Ji HZ, Shao JG, Bian ZL: High expression of ubiquitin-conjugating enzyme E2A predicts poor prognosis in hepatocellular carcinoma . Oncology letters 2018,15 (5):7362-7368.

9. Zhao Y, Alexandrov PN, Jaber V, Lukiw WJ: Deficiency in the Ubiquitin Conjugating Enzyme UBE2A in Alzheimer's Disease (AD) is Linked to Deficits in a Natural Circular miRNA-7 Sponge (circRNA; ciRS-7) . Genes 2016, 7 (12).

10. Ramatenki V, Potlapally SR, Dumpati RK, Vadija R, Vuruputuri U: Homology modeling and virtual screening of ubiquitin conjugation enzyme E2A for designing a novel selective antagonist against cancer. Journal of receptor and signal transduction research 2015, 35 (6):536-549.

11. Czeschik JC, Bauer P, Buiting K, Dufke C, Guillen-Navarro E, Johnson DS, Koehler U, Lopez-Gonzalez V, Ludecke HJ, Male A et al : X-linked intellectual disability type Nascimento is a clinically distinct, probably underdiagnosed entity . Orphanet journal of rare diseases 2013,8:146.

12. Ma D, Tan J, Zhou J, Zhang J, Cheng J, Luo C, Liu G, Wang Y, Xu Z: A novel splice site mutation in the UBE2A gene leads to aberrant mRNA splicing in a Chinese patient with X-linked intellectual disability type Nascimento . Molecular genetics \& genomic medicine 2019, 7 (11):e976. 
13. Yang Y, Haihong L, Jun Z, Min C, Ying L, Jinsheng H, Wei L, Jie Z, Xin N: The value of Gesell score in predicting the outcome of cochlear implantation in children .European archives of oto-rhinolaryngology : official journal of the European Federation of Oto-Rhino-Laryngological Societies 2017,274 (7):2757-2763.

14. Beckman L, Gustavson KH, Norring A: Finger and palm dermal ridge patterns in normal and mongoloid individuals (the Down syndrome) . Acta genetica et statistica medica 1962, 12 :20-27.

15. Budny B, Badura-Stronka M, Materna-Kiryluk A, Tzschach A, Raynaud M, Latos-Bielenska A, Ropers HH:Novel missense mutations in the ubiquitination-related gene UBE2A cause a recognizable X-linked mental retardation syndrome .Clinical genetics 2010, 77 (6):541-551.

16. Giugliano T, Santoro C, Torella A, Del Vecchio Blanco F, Bernardo P, Nigro V, Piluso G: UBE2A deficiency in two siblings: A novel splicing variant inherited from a maternal germline mosaicism . American journal of medical genetics Part A 2018, 176 (3):722-726.

17. Haddad DM, Vilain S, Vos M, Esposito G, Matta S, Kalscheuer VM, Craessaerts K, Leyssen M, Nascimento RM, Vianna-Morgante AM et al : Mutations in the intellectual disability gene Ube2a cause neuronal dysfunction and impair parkin-dependent mitophagy . Molecular cell 2013,50 (6):831-843.

18. Honda S, Orii KO, Kobayashi J, Hayashi S, Imamura A, Imoto I, Nakagawa E, Goto Y, Inazawa J:Novel deletion at Xq24 including the UBE2A gene in a patient with X-linked mental retardation . Journal of human genetics2010, 55 (4):244-247.

19. Nascimento RM, Otto PA, de Brouwer AP, Vianna-Morgante AM: UBE2A, which encodes a ubiquitin-conjugating enzyme, is mutated in a novel $\mathrm{X}$-linked mental retardation syndrome . American journal of human genetics 2006,79 (3):549-555.

20. Tolmacheva EN, Kashevarova AA, Nazarenko LP, Minaycheva LI, Skryabin NA, Lopatkina ME, Nikitina TV, Sazhenova EA, Belyaeva EO, Fonova EA et al : Delineation of Clinical Manifestations of the Inherited Xq24 Microdeletion Segregating with sXCI in Mothers: Two Novel Cases with Distinct Phenotypes Ranging from UBE2A Deficiency Syndrome to Recurrent Pregnancy Loss . Cytogenetic and genome research 2020,160 (5):245-254.

21. Tsurusaki Y, Ohashi I, Enomoto Y, Naruto T, Mitsui J, Aida N, Kurosawa K: A novel UBE2A mutation causes X-linked intellectual disability type Nascimento . Human genome variation 2017, $4: 17019$.

22. Wolanska E, Pollak A, Rydzanicz M, Pesz K, Klaniewska M, Rozensztrauch A, Skiba P, Stawinski P, Ploski R, Smigiel R: The Role of the Reanalysis of Genetic Test Results in the Diagnosis of Dysmorphic Syndrome Caused by Inherited xq24 Deletion including the UBE2A and CXorf56 Genes. Genes 2021,12 (3).

23. Arslan Satilmis SB, Kurt EE, Akcay EP, Sazci A, Ceylan AC: A novel missense mutation in the UBE2A gene causes intellectual disability in the large X-linked family. The journal of gene medicine 2021, 23 (2):e3307.

24. Hu H, Haas SA, Chelly J, Van Esch H, Raynaud M, de Brouwer AP, Weinert S, Froyen G, Frints $\mathrm{SG}$, Laumonnier $\mathrm{F}$ et al : X-exome sequencing of 405 unresolved families identifies seven novel intellectual disability genes . Molecular psychiatry 2016, 21 (1):133-148.

25. Tucker T, Zahir FR, Griffith M, Delaney A, Chai D, Tsang E, Lemyre E, Dobrzeniecka S, Marra M, Eydoux Pet al : Single exon-resolution targeted chromosomal microarray analysis of known and candidate intellectual disability genes . European journal of human genetics : EJHG 2014,22 (6):792-800. 

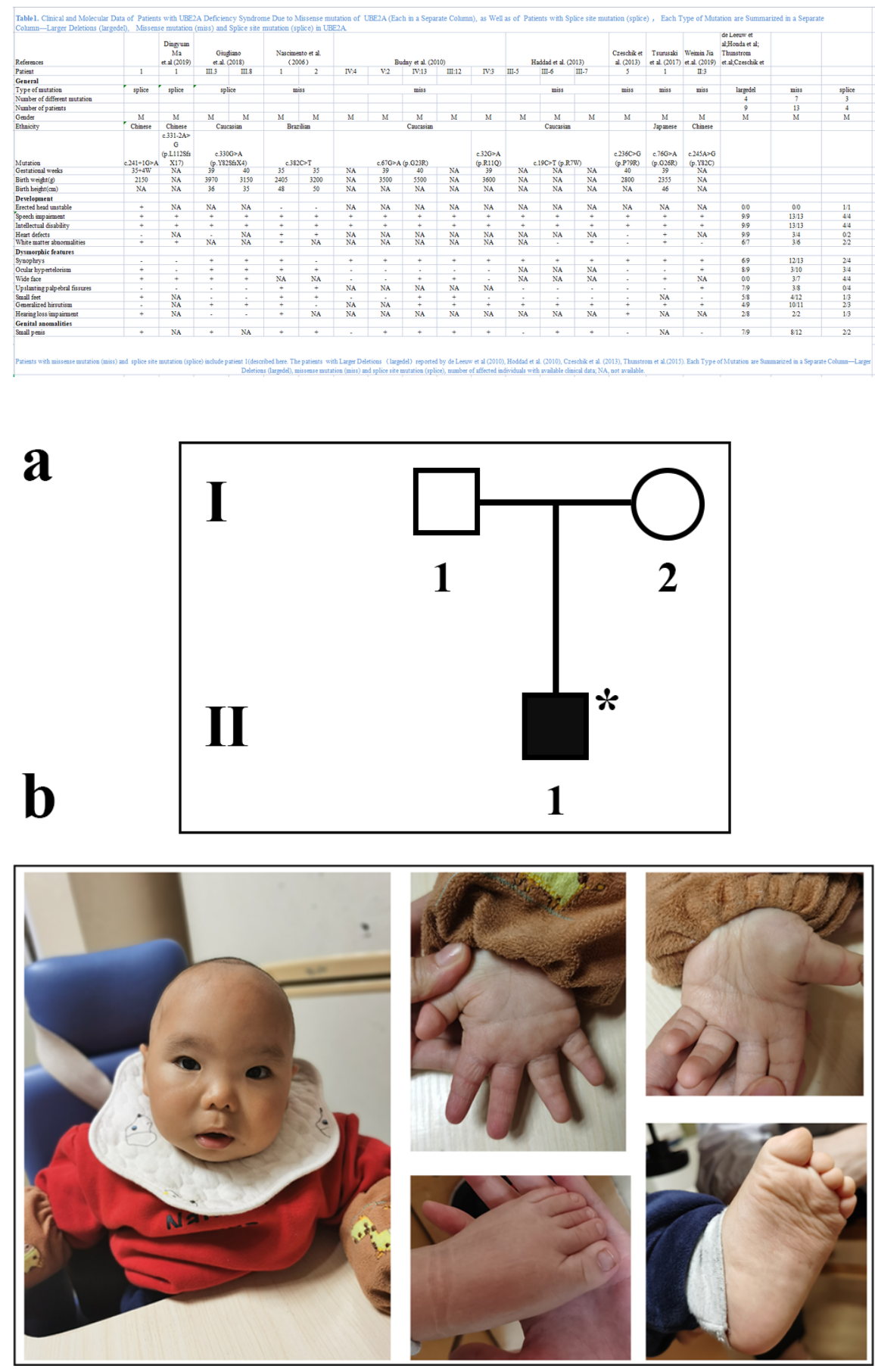
a

UBE2A:NM 003336.3:exon4:c. $241+1 \mathrm{G}>\mathrm{A}$ :

\begin{tabular}{|c|c|}
\hline Raference sequence & TGTTCCATCCAAATGGCAAGTATCACTTTTA \\
\hline $\begin{array}{l}\text { Sequence of the } \\
\text { proband }\end{array}$ & 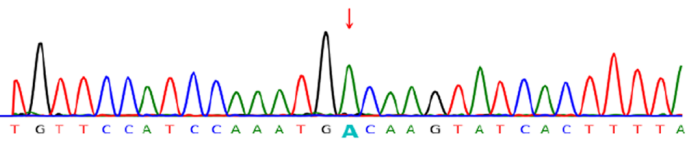 \\
\hline $\begin{array}{l}\text { Sequence of the } \\
\text { proband's father }\end{array}$ & 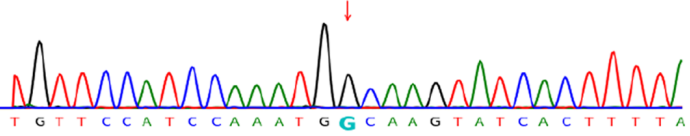 \\
\hline $\begin{array}{l}\text { Sequence of the } \\
\text { proband's mother }\end{array}$ & 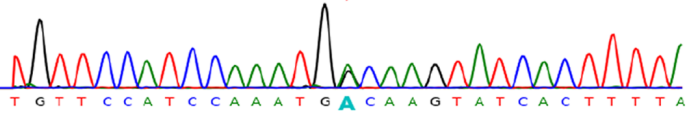 \\
\hline
\end{tabular}

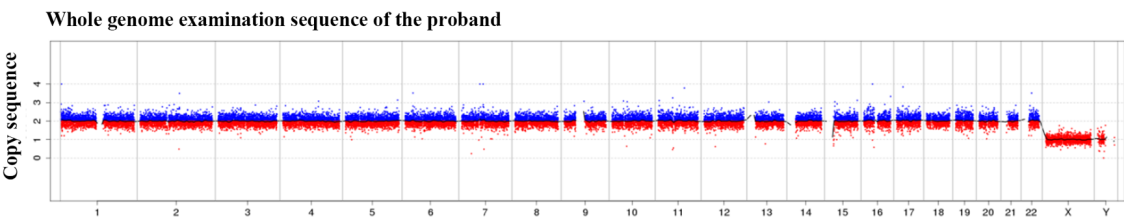

a

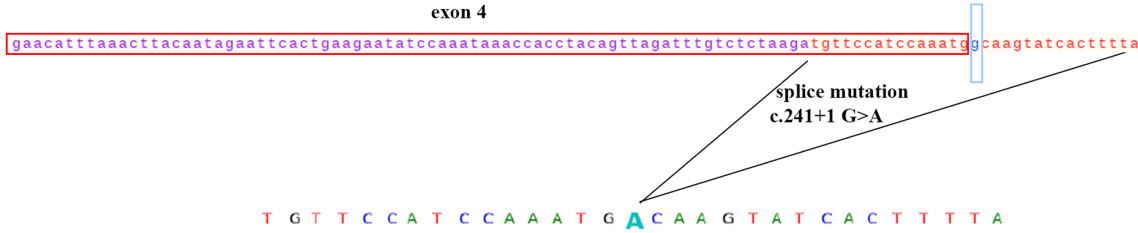

Sequence of the

proband

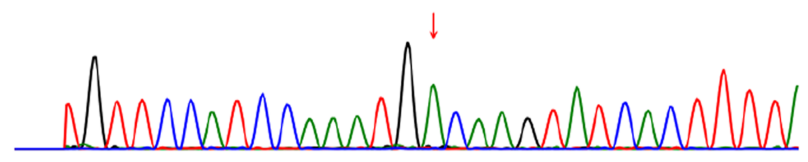

b

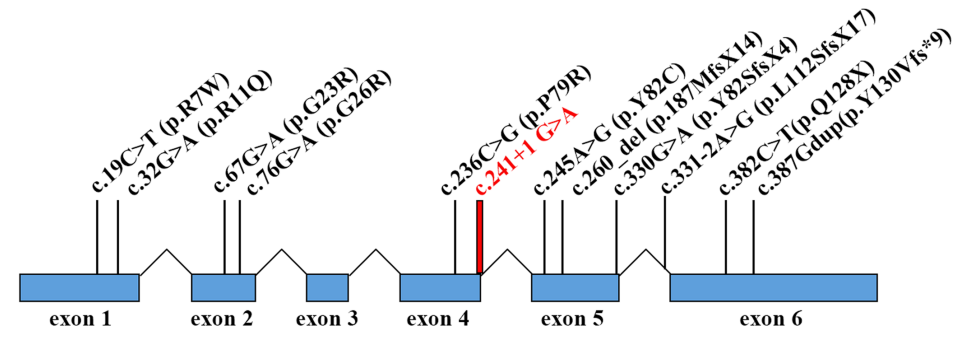




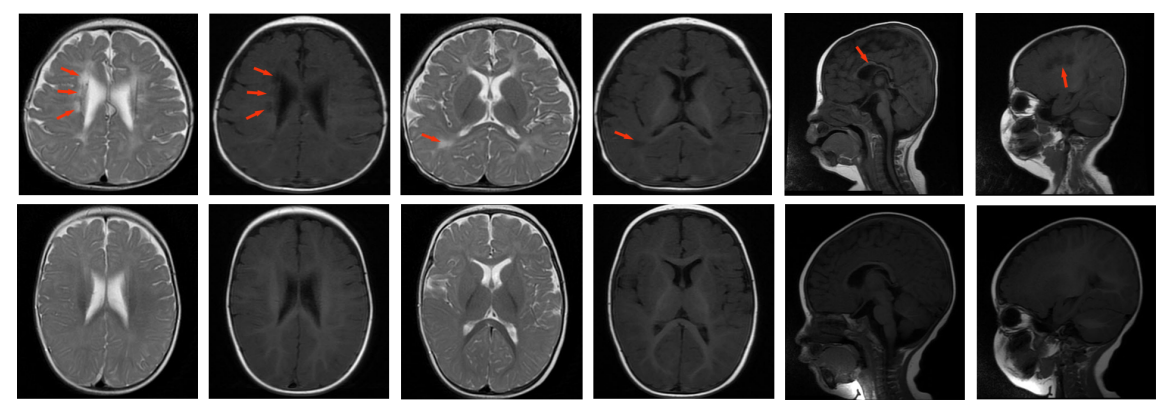

